\title{
Pre-treatment and continuous administration of simvastatin during sepsis improve metabolic parameters and prevent CNS injuries in survivor rats
}

\section{Carlos Henrique Rocha Catalão ( $\sim$ caique@usp.br)}

University of Sao Paulo Faculty of Medicine of Ribeirao Preto: Universidade de Sao Paulo Faculdade de Medicina de Ribeirao Preto https://orcid.org/0000-0003-2883-3103

\section{Anderson de Oliveira Souza}

Federal University of Mato Grosso: Universidade Federal de Mato Grosso

\section{Nilton Nascimento Santos-Junior}

University of Sao Paulo Faculty of Medicine of Ribeirao Preto: Universidade de Sao Paulo Faculdade de Medicina de Ribeirao Preto

\section{Luis Henrique Angenendt da Costa}

University of Sao Paulo Faculty of Medicine of Ribeirao Preto: Universidade de Sao Paulo Faculdade de Medicina de Ribeirao Preto

\section{Jonathas Rodrigo dos Santos}

University of Sao Paulo Faculty of Pharmaceutical Sciences of Ribeirao Preto: Universidade de Sao Paulo Faculdade de Ciencias Farmaceuticas de Ribeirao Preto

\section{Luciane Carla Alberici}

University of Sao Paulo Faculty of Pharmaceutical Sciences of Ribeirao Preto: Universidade de Sao Paulo Faculdade de Ciencias Farmaceuticas de Ribeirao Preto

\section{Maria José Alves Rocha}

University of Sao Paulo Faculty of Philosophy Sciences and Letters of Ribeirao Preto: Universidade de Sao Paulo Faculdade de Filosofia Ciencias e Letras de Ribeirao Preto

\section{Research Article}

Keywords: experimental sepsis, metabolism, metabolic disorders, HMG-CoA inhibitors, cytokines, glia

Posted Date: January 21st, 2022

DOI: https://doi.org/10.21203/rs.3.rs-1202746/v2

License: (9) This work is licensed under a Creative Commons Attribution 4.0 International License. Read Full License 


\section{Abstract}

Sepsis causes overproduction of inflammatory cytokines, organ dysfunction and cognitive impairment in survivors. In addition to inflammation, metabolic changes occur according to the stage and severity of the disease. Understanding the role and place of metabolic disturbances in the pathophysiology of sepsis is essential to evaluate the framework of septic patients, predict the syndrome progress and define treatment strategies. We investigated the effect of simvastatin on the disease time course and on metabolic alterations, especially with respect to their possible consequences in the CNS of surviving rats. The animals of this study were weighed daily and followed for 10 days to determine the survival rate. In the first experiment, control or CLP-animals were randomized in $24 \mathrm{~h}, 48 \mathrm{~h}$ and 10 days after septic induction, for bacterial load determination and, quantification of cytokines. In the second experiment, control or CLP-animals were treated or not with simvastatin and randomized in the same three time points for cytokines quantification and assessment of their body metabolism and locomotor activity (at $48 \mathrm{~h}$ and 10 days), as well as the evaluation of cytoarchitecture and astrogliosis (at 10 days). The CLPrats treated with simvastatin showed a reduction in plasma cytokines and improvement in metabolic parameters and locomotor activity, followed by minor alterations compatible with apoptosis and astrogliosis in the hippocampus and prefrontal cortex. These results suggest that the anti-inflammatory effect of simvastatin plays a crucial role in restoring energy production, maintaining a hypermetabolic state necessary for the recovery and survival of these CLP-rats.

\section{Introduction}

Sepsis has been the focus of intense investigation because of its clinical impact related to high mortality rates and associated morbidities that generate financial burden on the health care system [1]. Despite the intense and progressive effort in sepsis research, it is still considered a major health issue worldwide. Global data on sepsis incidence and mortality reported approximately 19.4 million cases and 5.3 million deaths annually [2]. With few exceptions, even developed countries present high mortality rates, leading to high costs (US\$24 billion annually, in the United States for example) $[3,4]$.

Due to the relevance of sepsis in clinical practice, the investigation of different aspects of sepsis pathophysiology through reliable experimental models is essential for the development new therapeutic approaches. Our research group has used the polymicrobial intra-abdominal infection model induced by cecal ligation and puncture (CLP) to investigate relevant aspects of sepsis, such as neuroendocrine and cognitive dysfunctions [5-7]. Using the CLP model, it is possible to modulate the severity of the ensuing infection by varying the amount and size of the needle used to puncture the cecum[ $[8,9]$. In this study, we used a well-established model of sepsis survivor animals to monitor the evolution of peripheral infection and its impact or consequences on the CNS. Particularly, we were interested in the effects of simvastatin in this context.

In the CLP model, there is a progressive release of pro-inflammatory cytokines that will play an essential role in activating the neuroendocrine response, mediating metabolic changes, and altering 
metabolism[10,11]. In the course of sepsis, these metabolic changes occur according to the stage and severity of the disease, with a dysregulation in the metabolism of all macronutrients - carbohydrates, lipids, and proteins[10, 12]. In this study, we use indirect calorimetry to analyze whole-body energy metabolism by measuring energy expenditure (EE) and identifying the substrate consumed through calculating the respiratory quotient $(\mathrm{RQ})[13]$. Understanding the role and place of metabolic disturbances in the pathophysiology of sepsis is essential for better treatment management in patients and for discovering targets for potential new therapies[14].

Considering these inflammatory and metabolic parameters, we used a statin as a pharmacological tool due to its known pleiotropic effects [15]. Several studies have shown that HMG-CoA reductase inhibitors, such as simvastatin, have anti-inflammatory properties that may play a role in modulating the immune system and contribute to the treatment of even neuroinflammatory diseases [6, 16-21]. This pleiotropic effect occurs in part through molecular mechanisms related to a cellular signaling cascade triggered by the inhibition of isoprenoid compounds and G-protein prenylation [22]. Given the wide use of statins by the world population and its anti-inflammatory effect, here we investigate the simvastatin effect on the time course of CLP-induced sepsis, by analyzing metabolic changes and histopathological assessments in the CNS.

\section{Material And Methods}

\section{Animals}

Male Wistar (280 - 360 grams) provided by the Central Animal Facility of the University São Paulo, Ribeirão Preto Campus, were housed in collective cages under controlled temperature $\left(25 \pm 1^{\circ} \mathrm{C}\right)$ and photoperiodic (12:12 h night:day cycle) conditions with water and commercial diet ad libitum (Nuvilab CR-1 Autoclavable, NUVITAL Nutrients S/A). The animals were weighed every day for 10 days. All experiments were carried out according to the National Council of Animal Experiment Control (CONCEA) and with approval by the Institutional Animal Care and Use Committee at the School of Dentistry of Ribeirão Preto, University of São Paulo (protocol number: \#2019.1.51.58.6). We used human endpoints in shock research [23] as criteria to euthanize cecal ligation and puncture (CLP) animals in high suffering, immediately before or soon after the studied time points [6].

\section{Cecal ligation and puncture (CLP) surgery and drug administration}

Polymicrobial sepsis was induced by CLP, as previously described $[5,18,24]$. This model has been widely used in our laboratory to investigate neuroimmunoendocrine alteration during sepsis. Briefly, animals were anesthetized with tribromoethanol (TBE) 2.5\% (Sigma- Aldrich) in saline solution $(250 \mathrm{mg} / \mathrm{kg}$ ) and an abdominal incision of $2 \mathrm{~cm}$ was performed followed by exposure of the cecum and obstruction (partial ligation) of the ileocecal valve. The cecum was punctured once with a $14 \mathrm{G}$ needle and after 
checking the extravasation of the fecal content, it was reintroduced into the peritoneal cavity and the incision was sutured. Sterile saline was applied subcutaneously as resuscitation fluid $(20 \mathrm{ml} / \mathrm{kg})$.

Simvastatin (trade name Zocor ${ }^{\circledR}$ - MSD, Merck Sharp \& Dohme, UK) was dissolved in sterile saline ( $\mathrm{NaCl}$ $0.9 \%$ ) and administered by gavage at a dose of $20 \mathrm{mg} / \mathrm{kg}$ in according to our previous study [18]. The animals were treated with simvastatin or an equivalent volume of saline 4 days before and $24 \mathrm{~h}, 48 \mathrm{~h}$, or 10 days after CLP surgery.

\section{Experimental protocol}

In the first experiment, control (naive = non-manipulated) or CLP-animals were randomized in three different time points ( $24 \mathrm{~h}, 48 \mathrm{~h}$, and 10 days after CLP-surgery), to bacterial load determination and cytokines quantification. In the second experiment, CLP-animals were treated with simvastatin and randomized in the same three-time points to cytokines quantification, body metabolism assessment by indirect calorimetry and locomotor activity (48 $\mathrm{h}$ and 10 days) and, histological and immunohistochemical evaluation (10 days). In both experiments, all the animals were weighed every day and followed for 10 days to determine the survival rate.

\section{Bacterial cultures}

Brain, spleen and mesenteric lymph node (MLN) samples were cultured at the time points $24 \mathrm{~h}, 48 \mathrm{~h}$, and 10 days after CLP-surgery [25]. Plates were incubated at $37^{\circ} \mathrm{C}$ in aerobic conditions for $48 \mathrm{~h}$, and then the number of colony-forming units (CFU)/mg for each organ or tissue was counted.

\section{Cytokines quantification}

Plasma IL-1 $\beta$ and IL- 6 concentrations were determined using specific enzyme-linked immunosorbent assay (ELISA) kits for each cytokine (R\&D Systems, Minneapolis, MN, USA) according to the manufacturer's instructions and as described in previous work of this laboratory [5]. To interrupt the reaction, we added to each well $25 \mu \mathrm{L}$ of $2 \mathrm{~N}$ sulfuric acid (H2SO4). The detection limits for IL-1 $\beta$ and IL- 6 specific ELISA kits were 5,10 , and $5 \mathrm{pg} / \mathrm{mL}$, respectively. The absorbance was measured at $450 \mathrm{~nm}$ in a microplate reader (Synergy ${ }^{\mathrm{TM}} \mathrm{H} 1$, BioTek ${ }^{\circledR}$ Instruments, Inc.).

\section{Indirect calorimetry and locomotor activity}

Analyses of the animals' body metabolism were performed at $48 \mathrm{~h}$ and 10 days after CLP-surgery by indirect calorimetry using the Oxylet Physiocage ${ }^{\mathrm{TM}}$ (PanLab, Barcelona, Spain) equipment. The experiment was carried in two metabolic boxes, with one animal per box, and recording for $14 \mathrm{~h}$, of which the final $12 \mathrm{~h}$ were analyzed. The following metabolic variables were considered: the volume of carbon dioxide produced $\left(\mathrm{VCO}_{2}\right)$, the volume of oxygen consumed $\left(\mathrm{VO}_{2}\right)$ and energy expenditure $(\mathrm{EE})$. The respiratory quotient $(\mathrm{RQ})$ calculated by the relation $\left[\mathrm{VCO}_{2} / \mathrm{VO}_{2}\right]$ was used for the estimation of macronutrient oxidation and locomotor activity (horizontal and vertical [rearing] movements).

\section{Histological and immunohistochemical assays}


The animals were perfused with $250 \mathrm{~mL}$ of PBS followed by $250 \mathrm{~mL}$ of fixative solution (4\% paraformaldehyde in $0.1 \mathrm{~mol} / \mathrm{L}$ phosphate buffer). Brains were post-fixed in paraformaldehyde (4\%) for 2 days at $4{ }^{\circ} \mathrm{C}$ and then kept in alcohol at $70 \%$. After stepwise dehydration and diaphanization, the brains were embedded in paraffin. Finally, the prefrontal cortex and hippocampus portions were coronally cut into $5 \mu \mathrm{m}$ thick sections and mounted on gelatin-coated slides. Histological assessment was performed by hematoxylin and eosin staining in order to assess the general cytoarchitecture, structure distribution, and cellular density. Histological changes were examined by a pathologist who was blinded to the identification of the treatment groups.

Immunohistochemical assays were used to detect reactive astrocytes. Sections were incubated overnight at $4^{\circ} \mathrm{C}$ with an anti-GFAP (glial fibrillary acidic protein) polyclonal primary antibody generated in rabbits (Z0334 DAKO, Glostrup, Denmark) diluted 1:6,000 in bovine serum albumin (BSA). Subsequently, the sections were incubated with a biotinylated goat anti-rabbit secondary antibody (Santa Cruz Biotechnology SC-2040) diluted 1:300 in BSA. Finally, the sections were incubated with HRP streptavidin reagent (dilution 1:400; Thermo Scientific JG 122591) and reacted with diaminobenzidine (DAB, Sigma D5905) for $1 \mathrm{~min}$. Staining specificity was checked by the omission of the primary antibody in some sections, resulting in the complete elimination of the immunoreaction signal.

Images were captured using an AxioCam MRc system (Zeiss) coupled to a Zeiss KS300 microscope. The anatomical description of brain regions was done according to the rat brain atlas of Paxinos and Watson [26].

\section{Statistical analysis}

All results are expressed as mean \pm standard error of the mean (SEM). Cytokines, weight gain, and locomotor activity results were analyzed by One-way analysis of variance (ANOVA). Indirect calorimetry results were analyzed by Two-way ANOVA. Both ANOVAs were followed by a Tukey's post-hoc test for multiple comparisons. The survival rate was analyzed by the Log-rank (Mantel-Cox) test. All statistical analyses were performed using GraphPad@Prism software, version 9.0 (San Diego, CA, USA). Variables

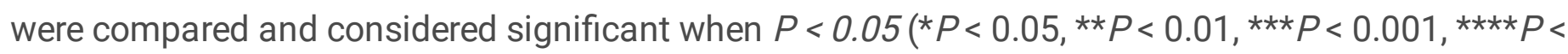
0.0001).

\section{Results}

Screening of sepsis severity through quantification of bacterial load and plasma cytokines during 10 days after CLP-surgery

The animals that underwent CLP-surgery showed typical signals of clinical sepsis, including lethargy, piloerection, and tachypnea. In this sepsis model, $50 \%$ of the animals died between 24 and 48 hours after CLP-surgery, with $30 \%$ at 48 hours. The mortality rate at the end of day 10 was $70 \%$ (Figure $1 \mathrm{~b}$ ).

At 24 and 48 hours, we observed statistically significant increase in the bacterial load present in the spleen (24 h: $P<0.01 ; 48$ h: $P<0.0001)$ and mesenteric lymph node (MLN) $(24 \mathrm{~h}: P<0.05 ; 48 \mathrm{~h}: P<$ 
0.0001) of septic animals compared to their controls. At 10 days after septic induction, no bacteria were found in any of the organs studied. No bacteria were found in the brain tissue at any of the time points studied (Figure 1c). We also found an increase in plasma cytokines at $24 \mathrm{~h}$ (IL-1 $\beta$ : $P<0.001$; IL-6: $P<$ $0.001)$ and $48 \mathrm{~h}(\mathrm{IL}-1 \beta: P<0.0001 ; \mathrm{IL}-6: P<0.0001)$ in septic animals compared to control animals. At day 10 days after septic induction, there was a reduction of these plasma cytokines to levels similar to those of the controls (Figure 1d).

At the critical time point of sepsis, both bacterial load (spleen: $P<0.001$; MLN: $P<0.01$ ) and cytokine levels (IL-1 $\beta$ : $P<0.001$; IL-6: $P<0.05$ ) were higher at $48 \mathrm{~h}$ when compared to the $24 \mathrm{~h}$ time point after CLP-surgery (Figure 1c, d).

\section{Analysis of survival curve and plasma levels of cytokines following simvastatin treatment}

Considering 24 and $48 \mathrm{~h}$ as the period with the highest number of animal deaths, and the highest cytokines plasma levels, the reduction in these levels indicate a simvastatin effect. Simvastatin treatment given 4 days before and 10 days after CLP-surgery (Figure 2a) showed a survival rate that was $30 \%$ higher compared to the untreated septic animals. Only at the $48 \mathrm{~h}$ time point we observed deaths in the treatment group (Figure $2 \mathrm{~b}$ ). Simvastatin treatment reduced the plasma levels of IL-1 $\beta(P<0.0001)$ and IL-6 $(P<0.001)$ cytokines, but only at the $48 \mathrm{~h}$ time point after sepsis induction (Figure $2 \mathrm{c})$. Additionally, the comparison of the simvastatin-treated groups for the two time points showed a decrease in the plasma levels of IL-1 $\beta(P<0.001)$ and IL-6 $(P<0.05)$ cytokines at 48 hours (Figure $2 \mathrm{~d}$ )

Measurement of metabolic parameters and locomotor activity at 48 hours and 10 days after CLP-surgery on simvastatin-treated rats

Forty-eight hours after CLP-surgery, septic animals showed a reduced energy metabolism $(P<0.0001)$ and locomotor activity $(P<0.01)$. Simvastatin treatment restored all these metabolic parameters as $\mathrm{VCO}_{2}$ $(P<0.0001), \mathrm{VO}_{2}(P<0.0001), \mathrm{RQ}(P<0.001)$ and $\mathrm{EE}(P<0.0001)$ compared to untreated septic rats (Figure 3b). Additionally, there was an increase in both horizontal movements $(P<0.05)$ and rearings $(P<$ 0.01) of these animals (Figure $3 c$ ).

Ten days after CLP-surgery, sepsis survivor animals showed an increase in some metabolic parameters, such as $\mathrm{VCO}_{2}(P<0.0001)$ and $\mathrm{RQ}(P<0.0001)$ (Figure 4c). However, there was a considerable reduction in body weight at the end of the experiment (Figure $4 \mathrm{~b})$. Simvastatin-treated animals remained with all metabolic parameters increased at the end of the experiment, but RQ values $(P<0.01)$ were lower when compared to untreated animals (Figure 4c). Simvastatin treatment restored body weight assessed at 10 days after sepsis induction $(P<0.01)$ (Figure $4 b)$. Finally, there was only an increase in rearing movement $(P<0.05)$ of these treated animals (Figure $4 \mathrm{~d})$. 
Plasma cytokines quantification and histopathological assessment in the prefrontal cortex and hippocampus 10 days after CLP-surgery on simvastatin-treated rats

There was no difference in the plasma levels of IL-1 and IL- 6 between the sepsis survivor groups studied (Figure 5b). In septic animals, the histopathological assessment in the prefrontal cortex and hippocampus (dentate gyrus and CA1 region) showed morphological characteristics suggestive of apoptosis, such as irregular cytoplasmic borders, in addition to nuclear fragmentation and condensation. These changes were less intense in septic animals treated with simvastatin (Figure $5 c, d, e$ - upper squares). Reactive astrocytes stained with GFAP were more evident in the septic animals, showing hypertrophic processes. In contrast, simvastatin-treated rats showed scattered astrocytes, with thinner astrocytic processes (Figure 5c, d, e - lower squares).

\section{Discussion}

Despite recent medical advances, the incidence of sepsis has increased, especially after data collection in regions with a lower Socio-demographic Index (SDI) [27]. The lack of knowledge about the evolution of the disease and the difficulty in providing an early diagnosis contribute to increase this trend and the mortality of patients $[28,29]$. Current therapeutic strategies for treating sepsis depend on antibiotics, fluid replacement, and symptomatic therapy [30]. Although there is no specific treatment for sepsis, several experimental studies have proposed potential new drugs or adjuvant therapies. In the present report, we show that the use of simvastatin 4 days before and 10 days after CLP-surgery improved metabolic parameters and prevented CNS changes in sepsis survivor animals.

Here, we show that at 24 and 48 hours after septic induction there is a progressive increase in bacterial load in the spleen and mesenteric lymph node of septic animals, coinciding with the time points of high mortality. Furthermore, we observed that bacterial dissemination increases in parallel with the increase of pro-inflammatory cytokines in the plasma of these animals. Recent studies have shown similar results by correlating severity and mortality scores with bacterial dissemination and cytokine levels in different organs in a pneumonia-induced sepsis model [31]. Although we did not adopt a score to assess sepsis severity, our animals showed typical signs, such as lethargy, piloerection, and tachypnea, which are parameters considered reliable to attest the severity of the disease [32,33]. Forty-eight hours after sepsis induction, the animals had a higher bacterial load in organs studied, higher plasma levels of proinflammatory cytokines, and a higher mortality rate. Other studies using the CLP model showed similar results, demonstrating that this appears to be the critical time point of sepsis in Wistar rats [24, 34].

The severity of sepsis has been linked to plasma cytokine concentrations for a long time [35]. Considering 24 and 48 hours as the period with the highest number of animal deaths, the action of simvastatin was analyzed by quantifying the plasma levels of cytokines. With the simvastatin administration, survival was $100 \%$ at 24 hours and $60 \%$ at 48 hours after sepsis induction, remaining unchanged up to 10 days. It is likely that this maintenance of survival rate is related to the considerable 
decrease in IL-1b and IL-6 levels observed at this time point in animals treated with simvastatin. One of the biggest challenges in controlling the progression of sepsis is to modulate the 'cytokine storm' in the early stages of the disease $[36,37]$. Cytokines are often responsible for triggering the inflammatory cascade and production of mediators such as nitric oxide, chemokines, and free radicals, leading to organ dysfunction and death $[38,39]$. Previous work has shown that this anti-inflammatory effect of simvastatin, by decreasing the overproduction of pro-inflammatory cytokines, was able to limit the release of other mediators, such as nitric oxide and reactive oxygen species, and to increase the survival of septic animals $[18,40]$. This pleiotropic effect of statins is extremely relevant, considering that several clinical and preclinical studies have used plasma levels of cytokines as surrogates for diagnosis and prognosis of organ dysfunction and mortality [37, 41-44].

Metabolic responses to sepsis are often difficult to measure, as they depend on several factors, including the body nutritional status and hormonal aspects $[45,46]$. Furthermore, the stage of disease during which the patient or animal is studied must be considered and may explain the variability of results reported in the literature $[47,48]$. In this work, we used indirect calorimetry to assess the energy metabolism in sepsis survivor rats treated with simvastatin. Our septic animals evaluated 48 hours after CLP-surgery had considerably lower calorimetric parameters $(R Q=0.90)$ compared to treated animals $(R Q=1.02)$. This discrepancy in the RQ values indicates that the animals of this experimental groups consume different substrates as an energy source, since higher RQ values suggest a predominant use of carbohydrates as an energy substrate [49]. Moreover, our results showed an increase in the measurement of EE and locomotor activity of septic animals treated with simvastatin compared to those not treated, suggesting a restoration of energy production. Taken together, these results lead us to infer that the simvastatin administration may have contributed to the production of a hypermetabolic state, necessary for the recovery of these animals. Several authors agree that the absence or premature terminus of a hypermetabolic state is associated with severe prognosis [48, 50,51]. Higher mortality was observed in septic animals that remained in a hypometabolic state and were unable to transition from lipid utilization to carbohydrates [50]. Similarly, preterminal patients have hypofunctional changes in metabolism, such as decreased oxygen consumption, triglyceride clearance, and hepatic mitochondrial activity $[47,52]$.

The inability to sustain a hypermetabolic state and the incapacity to transit to carbohydrate utilization is associated with a dysregulation of the energy metabolism caused by mitochondrial dysfunction [53]. Thus, it is possible to infer that the improvement in metabolic parameters and the restoration of energy production is related to the antioxidant action of statins [54,55]. Previous studies have shown that treatment with statins reduced glial activation and oxidative stress at 48 hours after sepsis induction [17, 18]. It is likely that this effect is related to the inhibition of the synthesis of isoprenoid compounds and prenylation of small $\mathrm{G}$ proteins. The occurrence of these events limits the production of inflammatory mediators and reactive oxygen species by negatively modulating the binding of these $G$ proteins to the plasma membrane to trigger the cell signaling cascade $[22,56]$.

Here, we observed that sepsis survivor animals evaluated 10 days after CLP-surgery showed an increase in RQ and EE values, suggesting success in the transition from lipid substrate to carbohydrates and 
reaching the hypermetabolic state. However, these animals were unable to restore body weight over the 10 days of evaluation. In experimental sepsis, the reduction in food and water consumption, even temporarily for $2-3$ days post-CLP, is sufficient to maintain the animals body weight below baseline values for 7 days [15]. In our study, it is likely that the severity of sepsis has produced a decrease in the search for food with consequent fasting and triggering of the mechanisms that promote lipolysis $[57,58]$. Although we have noticed an improvement in the horizontal movements of the untreated survivors, the number of vertical movements remained lower compared to the treated survivors. This can be explained by the attempt to minimize energy expenditure in face of foraging activities necessary for survival, inasmuch as vertical movements are costly $[59,60]$. The restoration of energy production at early time points provided simvastatin-treated rats with greater locomotor activity at both time points evaluated, in particular, an increase in vertical movements, observed even after 10 days of septic induction.

Patients that develop severe sepsis and septic shock have organ dysfunction and high levels of plasma cytokines, which can lead to death. Even with early intervention, patients who survive this condition are likely to develop neurocognitive impairments [35]. Cognitive deficits found in sepsis survivor animals are due to neurodegenerative processes associated with sustained neuroinflammation [6]. In this study, we performed morphological assessments in the hippocampus and prefrontal cortex, which are brain regions responsible for memory and cognition. We observed that animals surviving sepsis showed morphological characteristics suggestive of astrocytic activation and apoptosis. In sepsis, alterations in blood-brain barrier (BBB) permeability allow peripheral inflammatory mediators to reach the CNS [61,62]. These inflammatory mediators play a key role in sustained glial activation, which, in turn, contributes to the perpetuation of a neuroinflammatory environment with consequent cell death and cognitive impairment [6]. Our sepsis survivor animals did not show alterations in the plasma levels of IL- 1 and IL-6, demonstrating that, although peripheral inflammation has been resolved, glial activation persists up to 10 days after sepsis. Other authors have reported long-term brain dysfunction in surviving animals using the CLP model $[15,34,63]$.

Modulating sustained glial activation appears to be critical for containing persistent neuroinflammation in sepsis survivors [64-67]. Here, animals treated with simvastatin showed less noticeable morphological alterations in both investigated brain regions. Previous studies from our laboratory observed a decrease in Iba-1 expression accompanied by a reduction in cytokines and cleaved caspase-3, and an increase in $\mathrm{Bcl}-2$ in the brain of the simvastatin-treated animals. The likely mechanism proposed for this antiinflammatory effect of simvastatin is its role in the NF-KB (nuclear factor-kappaB)/SIRT1 (silent information regulator 1 ) signaling pathway by inhibiting the $M 1$ microglia phenotype [68-72]. Since this microglial activation induces the neurotoxic phenotype of astrocytes through the release of large amounts of cytokines, suppressing this activation may explain the decrease in astrogliosis observed in treated animals $[73,74]$.

In sepsis, although organ system dysfunction is more easily evaluated through routine laboratory tests, there are still no well-defined and practical biological markers in the CNS that can be targeted for therapeutic interventions. Therefore, studies that investigate the course of disease evolution and its 
relationship with the neuroinflammatory response are needed to clarify the brain dysfunction observed in sepsis survivors. Here we demonstrate that simvastatin administered 4 days before and 10 days after CLP-surgery improved metabolic parameters by sustaining a hypermetabolic state necessary for animal survival, reduced levels of pro-inflammatory cytokines, and prevented damage to areas responsible for memory and cognition of survivor rats. We believe that the pre-treatment was crucial, because in a pilot study we did not obtain satisfactory results when administering this drug only after septic induction. Other authors reported similar results using statins before and after sepsis [40]. As statins are widely used by the world population, further studies are needed to consider not interrupting this drug during the treatment of sepsis and to assess its likely potential as adjuvant neuroprotective therapy.

\section{Declarations}

\section{Acknowledgements}

The authors thank Nadir Fernandes for the technical support and Dr Klaus Hartfelder for his assistance with English language.

\section{Authors' contributions}

CHRC and MJAR conceived and designed research, with input from AOS, NNSJ and LHAC. CHRC, AOS, NNSJ, LHAC and JRS performed the experiments, analyzed data and drafting parts of the paper. CHRC, LCA and MJAR wrote the final manuscript and revised statistical analyses. All authors read and approved the final version of the manuscript.

\section{Funding}

The study was funded by the Fundação de Amparo à Pesquisa do Estado de São Paulo (FAPESP-grant 2017/12462-0) and the Coordenação de Aperfeiçoamento de Pessoal de Nível Superior - Brasil (CAPES) Finance Code 001.

\section{Availability of data and materials}

Data available on request from the authors.

\section{Conflict of interest}

The authors declare no conflict of interest.

\section{Ethical approval}

The study procedures were performed according to the National Council of Animal Experiment Control (CONCEA) and with approval by the Institutional Animal Care and Use Committee at the School of Dentistry of Ribeirão Preto, University of São Paulo (protocol number: \#2019.1.51.58.6). 


\section{Consent to participate}

All authors meet the qualifications for authorship and had an opportunity to read and comment the manuscript.

\section{Consent for publication}

All authors support publication of the manuscript in Molecular and Cellular Biochemistry.

\section{Authors' information}

Carlos Henrique Rocha Catalão, Email: caique@usp.br

Anderson de Oliveira Souza, Email: andersonosouza@uol.com.br

Nilton Nascimento Santos-Junior, Email: niltonneuro@gmail.com

Luis Henrique Angenendt da Costa, Email: luis.henrique.costa@usp.br

Jonathas Rodrigo dos Santos, Email: jonathas.rodrigo.santos@usp.br

Luciane Carla Alberici, Email: alberici@fcfrp.usp.br

Maria José Alves Rocha, Email: mjrocha@usp.br

\section{References}

1. Markwart R, Saito H, Harder T et al (2020) Epidemiology and burden of sepsis acquired in hospitals and intensive care units: a systematic review and meta-analysis. Intensive Care Med 46:1536-1551

2. Fleischmann C, Scherag A, Adhikari NK et al (2016) Assessment of Global Incidence and Mortality of Hospital-treated Sepsis. Current Estimates and Limitations. Am J Respir Crit Care Med 193:259-272

3. Liu V, Escobar GJ, Greene JD et al (2014) Hospital deaths in patients with sepsis from 2 independent cohorts. JAMA 312:90-92

4. Torio CM, Moore BJ (2016) National inpatient hospital costs: the most expensive conditions by payer, 2013.

5. Santos-Junior NN, Catalão CHR, Costa LHA et al (2018) Experimental sepsis induces sustained inflammation and acetylcholinesterase activity impairment in the hypothalamus. J Neuroimmunol 324:143-148

6. Catalão CHR, Santos-Junior NN, da Costa LHA et al (2020) Simvastatin Prevents Long-Term Cognitive Deficits in Sepsis Survivor Rats by Reducing Neuroinflammation and Neurodegeneration. Neurotox Res 38:871-886

7. Cazuza RA, Santos-Júnior NN, da Costa LHA et al (2020) Sepsis-induced encephalopathy impairs descending nociceptive pathways in rats. J Neuroimmunol 342:577198 
8. Rittirsch D, Huber-Lang MS, Flierl MA, Ward PA (2009) Immunodesign of experimental sepsis by cecal ligation and puncture. Nat Protoc 4:31-36

9. Ebong S, Call D, Nemzek J, Bolgos G, Newcomb D, Remick D (1999) Immunopathologic alterations in murine models of sepsis of increasing severity. Infect Immun 67:6603-6610

10. Englert JA, Rogers AJ (2016) Metabolism, Metabolomics, and Nutritional Support of Patients with Sepsis. Clin Chest Med 37:321-331

11. Molloy RG, Mannick JA, Rodrick ML (1993) Cytokines, sepsis and immunomodulation. Br J Surg 80:289-297

12. Irahara T, Sato N, Otake $\mathrm{K}$ et al (2018) Alterations in energy substrate metabolism in mice with different degrees of sepsis. J Surg Res 227:44-51

13. Speakman JR (2013) Measuring energy metabolism in the mouse - theoretical, practical, and analytical considerations. Front Physiol 4:34

14. Wasyluk W, Zwolak A (2021) Metabolic Alterations in Sepsis. J Clin Med 10:2412

15. Granger JI, Ratti PL, Datta SC, Raymond RM, Opp MR (2013) Sepsis-induced morbidity in mice: effects on body temperature, body weight, cage activity, social behavior and cytokines in brain. Psychoneuroendocrinology 38:1047-1057

16. Bu DX, Griffin G, Lichtman AH (2011) Mechanisms for the anti-inflammatory effects of statins. Curr Opin Lipidol 22:165-170

17. Reis PA, Alexandre PC, D'Avila JC et al (2017) Statins prevent cognitive impairment after sepsis by reverting neuroinflammation, and microcirculatory/endothelial dysfunction. Brain Behav Immun 60:293-303

18. Catalão CHR, Santos-Júnior NN, da Costa LHA, Souza AO, Alberici LC, Rocha MJA (2017) Brain Oxidative Stress During Experimental Sepsis Is Attenuated by Simvastatin Administration. Mol Neurobiol 54:7008-7018

19. Reis PA, Estato V, da Silva TI et al (2012) Statins decrease neuroinflammation and prevent cognitive impairment after cerebral malaria. PLoS Pathog 8:e1003099

20. Barone E, Cenini G, Di Domenico F et al (2011) Long-term high-dose atorvastatin decreases brain oxidative and nitrosative stress in a preclinical model of Alzheimer disease: a novel mechanism of action. Pharmacol Res 63:172-180

21. Tu Q, Cao H, Zhong W, Ding B, Tang X (2014) Atorvastatin protects against cerebral ischemia/reperfusion injury through anti-inflammatory and antioxidant effects. Neural Regen Res 9:268-275

22. Greenwood J, Steinman L, Zamvil SS (2006) Statin therapy and autoimmune disease: from protein prenylation to immunomodulation. Nat Rev Immunol 6(5):358-370

23. Nemzek JA, Xiao HY, Minard AE, Bolgos GL, Remick DG (2004) Humane endpoints in shock research. Shock 21:17-25 
24. Santos-Junior NN, Costa LHA, Catalão CHR, Kanashiro A, Sharshar T, Rocha MJA (2017) Impairment of osmotic challenge-induced neurohypophyseal hormones secretion in sepsis survivor rats. Pituitary 20:515-521

25. Opal SM, Palardy JE, Parejo N, Jasman RL (2003) Effect of anti-CD14 monoclonal antibody on clearance of Escherichia coli bacteremia and endotoxemia. Crit Care Med 31:929-932

26. Paxinos G, Watson C (2005) The Rat Brain in Stereotaxic Coordinates. Elsevier Academic Press

27. Rudd KE, Johnson SC, Agesa KM et al (2020) Global, regional, and national sepsis incidence and mortality, 1990-2017: analysis for the Global Burden of Disease Study. Lancet 395:200-211

28. Singer M, Deutschman CS, Seymour CW et al (2016) The Third International Consensus Definitions for Sepsis and Septic Shock (Sepsis-3). JAMA 315:801-810

29. Rubulotta FM, Ramsay G, Parker MM et al (2009) An international survey: Public awareness and perception of sepsis. Crit Care Med 37:167-170

30. Cohen J, Vincent JL, Adhikari NK et al (2015) Sepsis: a roadmap for future research. Lancet Infect Dis 15:581-614

31. Gonçalves MC, Horewicz VV, Lückemeyer DD, Prudente AS, Assreuy J (2017) Experimental Sepsis Severity Score Associated to Mortality and Bacterial Spreading is Related to Bacterial Load and Inflammatory Profile of Different Tissues. Inflammation 40:1553-1565

32. Shaver CM, Hauser AR (2004) Relative contributions of Pseudomonas aeruginosa ExoU, ExoS, and ExoT to virulence in the lung. Infect Immun 72:6969-6977

33. Machado GB, de Assis MC, Leão R et al (2010) ExoU-induced vascular hyperpermeability and platelet activation in the course of experimental Pseudomonas aeruginosa pneumosepsis. Shock 33:315321

34. Santos-Junior NN, Catalão CH, Costa LH et al (2018) Alterations in hypothalamic synaptophysin and death markers may be associated with vasopressin impairment in sepsis survivor rats. $\mathrm{J}$ Neuroendocrinol 1:e12604

35. Iwashyna TJ, Ely EW, Smith DM, Langa KM (2010) Long-term cognitive impairment and functional disability among survivors of severe sepsis. JAMA 304(16):1787-1794

36. Tisoncik JR, Korth MJ, Simmons CP, Farrar J, Martin TR, Katze MG (2012) Into the eye of the cytokine storm. Microbiol Mol Biol Re v 76:16-32

37. Gharamti A, Samara O, Monzon A et al (2021) Association between cytokine levels, sepsis severity and clinical outcomes in sepsis: a quantitative systematic review protocol. BMJ Open 11:e048476

38. Hotchkiss RS, Karl IE (2003) The pathophysiology and treatment of sepsis. N Engl J Med 348:138150

39. Fajgenbaum DC, June CH (2020) Cytokine Storm. N Engl J Med 383:2255-2273

40. Morel J, Hargreaves I, Brealey D et al (2017) Simvastatin pre-treatment improves survival and mitochondrial function in a 3-day fluid-resuscitated rat model of sepsis. Clin Sci (Lond) 131:747-758 
41. Damas P, Reuter A, Gysen P, Demonty J, Lamy M, Franchimont P (1989) Tumor necrosis factor and interleukin-1 serum levels during severe sepsis in humans. Crit Care Med 17:975-978

42. Michie HR, Manogue KR, Spriggs DR et al (1988) Detection of circulating tumor necrosis factor after endotoxin administration. N Engl J Med 318:1481-1486

43. Bozza FA, Salluh JI, Japiassu AM et al (2007) Cytokine profiles as markers of disease severity in sepsis: a multiplex analysis. Crit Care 11:R49

44. van den Berg S, Laman JD, Boon L et al (2013) Distinctive cytokines as biomarkers predicting fatal outcome of severe Staphylococcus aureus bacteremia in mice. PLoS One 8:e59107

45. Polito A, Sonneville R, Guidoux C et al (2011) Changes in CRH and ACTH synthesis during experimental and human septic shock. PLoS One 6:e25905

46. Mogensen KM, Robinson MK, Casey JD et al (2015) Nutritional Status and Mortality in the Critically III. Crit Care Med 43:2605-2615

47. Siegel JH, Cerra FB, Coleman B et al (1979) Physiological and metabolic correlations in human sepsis. Invited commentary. Surgery 86:163-193

48. Giovannini I, Boldrini G, Castagneto M et al (1983) Respiratory quotient and patterns of substrate utilization in human sepsis and trauma. JPEN J Parenter Enteral Nutr 7:226-230

49. Even PC, Nadkarni NA (2012) Indirect calorimetry in laboratory mice and rats: principles, practical considerations, interpretation and perspectives. Am J Physiol Regul Integr Comp Physiol 303:R459476

50. Li A, Mukhopadhyay A (2020) Substrate utilization and energy expenditure pattern in sepsis by indirect calorimetry. Crit Care 24:535

51. Fried RC, Bailey PM, Mullen JL, Stein TP, Crosby LO, Buzby GP (1986) Alterations in exogenous substrate metabolism in sepsis. Arch Surg 121:173-178

52. Cerra FB, Siegel JH, Coleman B, Border JR, McMenamy RR (1980) Septic autocannibalism. A failure of exogenous nutritional support. Ann Surg 192:570-580

53. Leverve XM (2007) Mitochondrial function and substrate availability. Crit Care Med 35:S454-460

54. Wagner AH, Köhler T, Rückschloss U, Just I, Hecker M (2000) Improvement of nitric oxide-dependent vasodilatation by HMG-CoA reductase inhibitors through attenuation of endothelial superoxide anion formation. Arterioscler Thromb Vasc Biol 20:61-69

55. Wassmann S, Laufs U, Bäumer AT et al (2001) Inhibition of geranylgeranylation reduces angiotensin II-mediated free radical production in vascular smooth muscle cells: involvement of angiotensin AT1 receptor expression and Rac1 GTPase. Mol Pharmacol 59:646-654

56. Liao JK, Laufs U (2005) Pleiotropic effects of statins. Annu Rev Pharmacol Toxicol 45:89-118

57. Wischmeyer PE, San-Millan I (2015) Winning the war against ICU-acquired weakness: new innovations in nutrition and exercise physiology. Crit Care 19(Suppl 3):S6

58. Arabi YM, Aldawood AS, Solaiman O (2015) Permissive Underfeeding or Standard Enteral Feeding in Critical Illness. N Engl J Med 373:1175-1176 
59. Jovalekic A, Hayman R, Becares N et al (2011) Horizontal biases in rats' use of three-dimensional space. Behav Brain Res 222:279-288

60. Jedidi-Ayoub S, Mishchanchuk K, Liu A, Renaudineau S, Duvelle É, Grieves RM (2021) Volumetric spatial behaviour in rats reveals the anisotropic organisation of navigation. Anim Cogn 24:133-163

61. Barichello T, Generoso JS, Collodel A, Petronilho F, Dal-Pizzol F (2021) The blood-brain barrier dysfunction in sepsis. Tissue Barriers 9:1840912

62. Dal-Pizzol F, Rojas HA, dos Santos EM et al (2013) Matrix metalloproteinase-2 and metalloproteinase- 9 activities are associated with blood-brain barrier dysfunction in an animal model of severe sepsis. Mol Neurobiol 48:62-70

63. Singer BH, Newstead MW, Zeng X et al (2016) Cecal Ligation and Puncture Results in Long-Term Central Nervous System Myeloid Inflammation. PLoS One 11:e0149136

64. Zrzavy T, Höftberger R, Berger T et al (2019) Pro-inflammatory activation of microglia in the brain of patients with sepsis. Neuropathol Appl Neurobiol 45:278-290

65. Michels M, Abatti MR, Ávila P et al (2020) Characterization and modulation of microglial phenotypes in an animal model of severe sepsis. J Cell Mol Med 24:88-97

66. Michels M, Abatti M, Vieira A et al (2020) Modulation of microglial phenotypes improves sepsisinduced hippocampus-dependent cognitive impairments and decreases brain inflammation in an animal model of sepsis. Clin Sci (Lond) 134:765-776

67. Yan C, Ma Z, Ma H et al (2020) Mitochondrial Transplantation Attenuates Brain Dysfunction in Sepsis by Driving Microglial M2 Polarization. Mol Neurobiol 57:3875-3890

68. Tian M, Qingzhen L, Zhiyang Y et al (2019) Attractylone attenuates sepsis-associated encephalopathy and cognitive dysfunction by inhibiting microglial activation and neuroinflammation. J Cell Biochem. doi:10.1002/jcb.27983

69. Pan S, Wu Y, Pei L et al (2018) BML-111 Reduces Neuroinflammation and Cognitive Impairment in Mice With Sepsis via the SIRT1/NF-kB Signaling Pathway. Front Cell Neurosci 12:267

70. Zhuo Y, Zhang S, Li C, Yang L, Gao H, Wang X (2018) Resolvin D1 Promotes SIRT1 Expression to Counteract the Activation of STAT3 and NF-KB in Mice with Septic-Associated Lung Injury. Inflammation 41:1762-1771

71. Lu D, Shen L, Mai H et al (2019) HMG-CoA Reductase Inhibitors Attenuate Neuronal Damage by Suppressing Oxygen Glucose Deprivation-Induced Activated Microglial Cells. Neural Plast 2019:7675496

72. Liu H, Yang J, Wang K, Niu T, Huang D (2019) Moderate- and Low-Dose of Atorvastatin Alleviate Cognition Impairment Induced by High-Fat Diet via Sirt1 Activation. Neurochem Res 44:1065-1078

73. Arranz AM, De Strooper B (2019) The role of astroglia in Alzheimer's disease: pathophysiology and clinical implications. Lancet Neurol 18:406-414

74. Liddelow SA, Guttenplan KA, Clarke LE et al (2017) Neurotoxic reactive astrocytes are induced by activated microglia. Nature 541:481-487 


\section{Figures}

\section{Figure 1}

Analysis of the evolution of sepsis induced by cecal ligation and puncture (CLP) during 10 days after surgery. Experimental protocol detailing the time points studied as $24 \mathrm{~h}, 48 \mathrm{~h}$ and 10 days (a). Septic animals presented mortality of $70 \%$ over a span of 10 days and the crucial death time point occurred at 48 hours (30\%) (b). At this same time point, there was a significant increase in bacterial load (c) and IL-1 $\beta$ and IL-6 cytokines (d). Ten days after CLP-surgery, there was sepsis recovery, as demonstrated by the absence of bacteria (c) and normalization of plasma levels of cytokines (d). Bars indicate mean \pm SEM ( $n$ = 5-8 animals per group). One-way ANOVA with Tukey's Multiple Comparison Test correction.

\section{Figure 2}

Effect of treatment with simvastatin $(20 \mathrm{mg} / \mathrm{kg}$, p.o.) 4 days before and $24 \mathrm{~h}, 48 \mathrm{~h}$ and 10 days after CLPsurgery on proinflammatory plasma cytokine levels and survival rate. Experimental protocol detailing the time points studied (a). Simvastatin-treated rats showed survival of $60 \%$ after 10 days CLP-surgery (b). Sepsis caused an increase in IL-1 $\beta$ and IL- 6 levels in the plasma. Simvastatin treatment prevented the increase of these cytokines only at $48 \mathrm{~h}$ (c). At this same time point, there was a significant reduction in these proinflammatory cytokines when compared with $24 \mathrm{~h}$ time point $(\mathrm{d})$. Bars indicate mean \pm SEM $(n=$ 5-8 animals per group). One-way ANOVA with Tukey's Multiple Comparison Test correction.

\section{Figure 3}

Effect of treatment with simvastatin (20 mg/kg, p.o.) 4 days before and $48 \mathrm{~h}$ after CLP-surgery on body metabolism and locomotor activity. Experimental protocol (a). Sepsis caused a decrease in all the metabolic parameters analyzed. Simvastatin administration caused an increase of body metabolism in the treated rats by elevation these parameters (b). Septic rats showed a decrease in locomotor activity while simvastatin-treated animals had an increase in both horizontal and vertical movements (c). Bars indicate mean \pm SEM ( $n=5$ animals per group). One-way ANOVA with Tukey's Multiple Comparison Test correction.

\section{Figure 4}


Effect of treatment with simvastatin (20 mg/kg, p.o.) 4 days before and 10 days after CLP-surgery on body metabolism, locomotor activity and weight. Experimental protocol (a). There was a considerable reduction in body weight of the sepsis survivor animals at the end of the experiment and simvastatin recovered the weight of the treated rats (b). Simvastatin-treated animals remained with all metabolic parameters increased at the end of the experiment except for the RQ values that were lower compared to untreated animals (c). There was only an increase in vertical movements (rearing) of these treated animals (d). Bars indicate mean \pm SEM ( $n=5$ animals per group). One-way ANOVA with Tukey's Multiple Comparison Test correction.

\section{Figure 5}

Effect of treatment with simvastatin ( $20 \mathrm{mg} / \mathrm{kg}$, p.o.) 4 days before and 10 days after CLP-surgery on proinflammatory plasma cytokine levels and histopathological alterations. Experimental protocol (a). There was no difference in levels of plasma of IL-1 $\beta$ and IL- 6 cytokines in any of the groups (b). Photomicrographs of different regions of rat brains stained for hematoxylin-eosin (HE) (upper squares) and immunostained for GFAP (lower squares): prefrontal cortex (c), dentate gyrus (d) and CA1 region (e) of the hippocampus. In septic animals, HE-stained brain regions showed cellular morphology with characteristics suggestive of apoptosis and astrocytes GFAP-immunostained with hypertrophic processes. In contrast, simvastatin-treated rats showed discrete morphological alterations and scattered astrocytes with thin astrocyte processes. Objective magnification: 40× (oil immersion). Scale bar, $20 \mu \mathrm{m}$. 